\section{Die Bedeutung des Pantopons (Sahli) für die Gynäkologie und Geburtshilfe.}

\section{Von Dr. Ernst Gräfenberg in Berlin.}

Nach der Einreihung des Sahlischen Opiumpräparates Pantopon (F. Hoffmann, La Roche \& Co.) in den Arzneischatz ist es das Bestreben gewesen, die Indikationen seiner Verwendung abzugrenzen. Dem allgemein gehaltenen Vorschlag Sahlis, ${ }^{1}$ ) das Pantopon in der gleichen Weise wie das Opium und Morphium zu verwenden, folgten bald Untersuchungen, die seine Verwendbarkeit für bestimmte Krankheitsgebiete in Erwägung zogen.

Die klinischen und experimentellen Beobachtungen von Sahli ${ }^{1}$ ), Rodari ${ }^{2}$ ), Heimann ${ }^{3}$ ) und Hallervorden ${ }^{4}$ ) haben gelehrt, daß das Präparat in jeder Weise dem Opium und Morphium gleichwertig ist, wenn es gilt, Schmerzen zu stillen, Reizzustände, psychische Aufregungszustände und Schlaflosigkeit zu bekämpfen. Deshalb hat auch Heimann der Verwendung des Pantopons in der Nachbehandlung der Operierten das Wort geredet und besonders hervorgehoben, daß es bei gutem Einfluß auf die Schmerzen die Darmtätigkeit weniger ungünstig beeinflußt als das Morphium und so den Abgang der Flatus nicht stört.

Das Pantopon enthält nach den Angaben Sahlis sämtliche Komponenten des Opiums. Es ist ein Gemisch der an Salzsäure gebundenen Opiumalkaloide in wasserlöslicher Form.

Neuere Studien pharmakologischer Wirkungen haben gelehrt, daß eine Mischung kleiner Mengen und ihre wiederholte Einführung in den Organismus eine Summation von Effekten hervorzurufen vermag, die nicht einer einfachen Addition gleichkommt. Diese experimentellen Erfahrungen bestätigen die empirische Tatsache, daß die Rohdroge mit ihren zahlreichen verschiedenen Komponenten wirksamer ist als alle künstlichen Alkaloidderivate.

Das Summationsgesetz der kumulierenden Wirkung kleiner polyvalenter Gemische der Narcotica ist durch $B$ ürgi ${ }^{5}$ ) exalkt bewiesen worden. Gleichzeitig oder auch kurz nacheinander eingeführte Narcoticagemische vermögen nach den schönen Untersuchungen dieses Forschers eine weit stärkere Wirkung zu entfalten, als die einfache Addition der Einzelwirkungen verlangen dürfte. Nach Bürgi beruht diese Eigentümlichkeit auf zellularphysiologischen Korrelationen des Zellindividuums gegenüber differenten Substanzen, die an die Zelle herantreten. Die Zelle hat für jede pharmakologisch wirksame Substanz einen spezifischen Rezeptor, der nach seiner Absättigung durch die zugeführte Lösung meist nicht in der Lage ist, von der gleichen Substanz mehr auf ${ }^{f}$ zunehmen. Er bedarf vielmehr einer gewissen Erholung, während der die Zelle entgiftet wird. Erst nach einem bestimmten Zeitintervall findet eine von neuem zugeführte gleiche Substanz wieder Angriffspunkta an dem gleichen Rezeptor. Da die Zelle zahlreiche Rezeptorenkomplexe besitzt, die für die ve:schiedenartigsten Substanzen bestimmt sind, so kann die Rezeptorengruppe für die eine Komponente völlig verbraucht sein, wenn für eine zweite differente Substanz noch alle Rezeptoren aufnahmefähig sind.

Nach dieser Theorie Bürgis wird die Mischnarkose das Narkosenideal darstellen, bei der die verschiedenen Narcotica gleichzeitig an den verschiedenen Rezeptoren der Zelle angreifen können. Die Narkosenempirie hat schon vor der Begründung durch die wissenschaftlichen Experimente den richtigen Weg beschritten, als sie durch die geschickte Kombination des Morphiums und Skopolamins mit dem Chloroform und dem Aether eine Mischnarkose schuf. (Hartog. $\left.)^{6}\right)$

1) Sahli, Therapeutische Monatshefte 1909. -2 ) Rodari, Thera. peutische Monatshefte 1909. - "3) Heiman n, Münchener medizinische Wochenschrift 1910, No. 7. - Hallervorden, Therapie der Gegenwart 1910, H. 5. - s) B ürgi, Deutsche medizinische Wochenschrift 1910, No. 1 u. 2. - $\left.{ }^{8}\right)$ Hartog, Münchener medizinische Wochenschrift 1903 .
Im Gegensatz zu diesen künstlich geschaffenen Kombinationen ist das Opium, die Rohdroge, ein Mittel, das in sich die polyvalentesten Narcotica vereinigt und deshalb ein natürliches Kombinationsnarkotikum darstellt. Hierauf beruht seine auch in der Gynäkologie immer noch unübertroffene Wirkung der Schmerzlinderung. Für die Allgemeinnarkose ist es dagegen nur wenig benutzt worden, denn seine Wirkung tritt bei gewöhnlicher Zufuhr vom Darm aus nicht schnell genug ein, und die bisher bekannten Opiumpräparate sind zur subkutanen Injektion ungeeignet.

Erst jetzt, wo es Sahli gelungen ist, in dem Pantopon ein für subkutane Injektionen umgewandeltes Opium herzustellen, hat seine Anwendung für narkotische Zwecke eine größere Ausdehnung erfahren können. Eine vollständige Narkose, in der größere Operationen ausgeführt werden könnten, läßt sich allerdings nicht erzielen. Seine Toleranzbreite ist nicht unbeschränkt, deshalb scheitern alle Versuche an der gleichen Klippe wie die jetzt fast völlig verlassene reine Morphium-Skopolaminnarkose v. Korffs. Wir erwarten eben nur eine Unterstützung der Inhalationsnarkose von diesen Injektionen. Die Beschränkung ihrer Verwendung auf dieses Gebiet hat dem Morphium-Skopolamin seine ausgedehnte Verbreitung verschafft.

Trotz der guten Erfahrungen, die mit den vorbereitenden Morphium-Skopolamin-Injektionen gemacht werden, ist doch stets die beschränkte Haltbarkeit des Skopolamins und seine schnelle Zersetzlichkeit in der Mischung mit dem Morphium eine unheilvolle Gefahr für den Praktiker gewesen. Dieser Faktor spielt besonders dann eine große Rolle, wenn die Lösungen nicht so häufig gebraucht und verbraucht werden wie im Großbetrieb der Kliniken, in denen eine Erneuerung der Lösungen immer schon nach Ablauf weniger Tage nötig wird.

Ich habe deshalb versucht, das künstliche Kombinationsnarkotikum Morphium-Skopolamin durch die natürliche und gebrauchsfertige Mischung der Opiumalkaloide des Pantopons zu ersetzen, dessen narkotischer Effekt bereits durch die Untersuchungen von Sahli und Rodari dargetan war.

Die unter Pfannenstiel ${ }^{1}$ ) an der Kieler UniversitätsFrauenklinik geübte Narkose bestand in der Aethernarkose mit der Wanscher-Grossmannschen Maske, die durch eine $1 \frac{1}{2}$ Stunden vorher ausgeführte Injektion von 0,01 Morphinum hydrochloricum $+0,0003$ Scopolamin: hydrobromicum eingeleitet wurde und der $1 / 2$ Stunde vor Beginn der Narkose die gleiche Dosis Skopolamin folgte. Die Nachtruhe vor der Operation sicherte $0,5 \mathrm{~g}$ Veronal.

In diesem Narkosenschema haben wir das Morphium bzw. das Morphium-Skopolamin durch Pantopon ersetzt.

Um ein kritisches Urteil über die Wirksamkeit der injizierten Substanzen fällen zu können, wurde bei allen Narkosen der Zeitpunkt des Erlöschens der Kornealreflexe als Vergleichspunkt bestimmt. Ein gut Teil der Beobachtungen erfreute sich der Unterstützung von Frau Dr. J. Hoeber (Kiel).

Die Zeitdauer vom Beginn der Narkose bis zum Stadium der Reflexlähmung ist verschieden je nach den zur Narkose benutzten Narcotica. Sie ist am längsten, wenn die Patienten ohne vorhergeschickte Injektion nur mit Aether betäubt würden. Die Beeinflussung dieses Stadiums durch die Injektion von Morphium, Skopolamin und Pantopon veranschaulicht die beigefügte Tabelle. Da unsere Beobachtungen ausschließlich bei Aethernarkosen mit der Wanscher-Grossmannschen Maske ausgeführt wurden, konnten die Schwankungen des Aetherverbrauches nicht exakt genug gemessen werden. Sie sind deshalb unberücksichtigt geblieben.

\begin{tabular}{|c|c|}
\hline Injektion vor der Operation & Kornealreflex erloschen \\
\hline 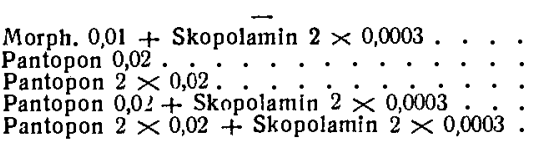 & $\begin{array}{c}\text { nach } 16.8 \text { Min. } \\
" \quad 14,6 n \\
" \quad 14,4= \\
" \quad 4-5 n \\
" \quad 12.6=0 \\
" \quad 4-6 n\end{array}$ \\
\hline
\end{tabular}
1903.

1) Pfannenstiel und Kroemer, Zentralblatt für Gynäkologie 
Der Einfluß der vorhergehenden Injektionen auf den Zeitpunkt der Areflexie ist ganz augenscheinlich. Der Kornealreflex erlischt fast fünf Minuten früher, wenn der Aetherinhalation eine Morphium (0,01)-Skopolamin $(2 \times 0,0003)$ Injektion vorausgeschickt wird. Dieser vorbereitenden Injektion von Morphium-Skopolamin kommt hinsichtlich ihrer Wirksamkeit eine einmalige Injektion von $0,02 \mathrm{~g}$ Pantopon, d. i. $1 \mathrm{ccm}$ der fertigen, $2 \%$ igen Pantoponlösung gleich. Denn 14,4 Minuten nach Beginn der Aethernarkose erlischt der Kornealreflex. Der Eintritt der Areflexie wird abermals um zwei Minuten beschleunigt, wenn einmal Pantopon $(0,02)$ und zweimal Skopolamin $(2 \times 0,0003 \mathrm{~g})$ mit einem der MorphiumSkopolamin-Technik entlehnten einstündigen Intervall injiziert werden.

Am schnellsten erlöschen die Kornealreflexe, wenn wir vor der Operation zweimal 0,02 Pantopon entsprechend der Morphium-Skopolamintechnik injizieren. Denn jetzt tritt das Narkosenoptimum bereits vier bis fünf Minuten nach Beginn der Aethernarkose ein. Es verstreicht nach einer zweimaligen Injektion des Pantopons bis zum Schwinden der Kornealreflexe nur ein Drittel der für die anderen Injektionen erforderlichen Zeit. Dieses eigenartige Verhältnis besteht sogar auch gegenüber der narkotischen Wirkung des MorphiumSkopolamins, dessen Narkosenoptimum um 14,6 Minuten auffällig kontrastiert gegen das nur fünf Minuten dauernde Stadium der Narkoseneinleitung mit dem Pantopon.

Daß für eine Narkose, die schon nach fünf Minuten ihr Optimum erreicht, weit weniger Aether nötig ist als bei einer das Dreifache erfordernden Technik, bedarf wohl keiner Begründung.

Nach diesen zweimaligen, durch ein einstündiges Intervall getrennten Pantoponinjektionen wurden keine Schädigungen beobachtet. Atmung und Puls bleiben unbeeinflußt, auch ist eine vermehrte Brechreizung nicht zu konstatieren.

Durch eine Kombination der zweimaligen Pantoponinjektionen $(2 \times 0,02)$ mit den üblichen Skopolamingaben $(2 \times 0,0003 \mathrm{~g})$ hat eine weitere Beschleunigung des Narkoseneintritts nicht erzielt werden können. Auch jetzt vergehen bis zum Erlöschen der Kornealreflexe noch vier bis sechs Minuten. Es bietet deshalb der Skopolaminzusatz gegenüber der beschriebenen Pantoponmethode keine Vorzüge. Zudem erwächst durch eine häufig nötige Anschaffung der leicht verderbenden Skopolaminlösung eine nicht unbeträchtliche Verteuerung. Die Herstellung einer fertigen Pantopon-Skopolaminlösung scheitert an der leichten Zersetzbarkeit des Skopolamins. Die Versuche mit diesem Gemisch wurden aufgegeben, als zwei Patienten nach der Narkose kollabierten. Die Ursache des Kollapses sind vermutlich die Zersetzungsprodukte des Skopolamins, von denen das Apoatropin mit Hilfe der Kaliumpermanganatprobe nach Kessel an seiner Braunfärbung leicht nachgewiesen werden konnte.

Es ist deshalb ratsamer, nur Pantopon ohne Vermischung mit Skopolamin zu injizieren. Je $1 \mathrm{ccm}$ der fertigen $2 \%$ igen Lösung wird $1 \frac{1 / 2}{b z w}$. $1 / 2$ Stunde vor Beginn der Narkose subkutan eingespritzt. Wird die Lösung nicht häufig gebraucht, so empfiehlt sich die Verwendung der von der Fabrik (Hoffmann, La Roche \& Co.) in den Handel gebrachten sterilisierten Ampullen mit $1 \mathrm{ccm}$ Inhalt. Für die Kliniken, wo zahlreiche Operationen häufige Injektionen erfordern, ist die Fläschchenpackung à $10 \mathrm{ccm}$ geeigneter.

Die augenfällige Verschiebung der Reizschwelle gab uns Veranlassung, das Pantopon auch in der Geburtshilfe zu prüfen. Zur Linderung der Wehenschmerzen unter der Geburt wurde pro dosi $1 \mathrm{ccm}$ der $2 \%$ igen Lösung $(0,02 \mathrm{~g}$ Pantopon) injiziert. Bei Mehrgebärenden mit ihrem abgekürzten Geburtsverlauf hat eine einmalige Injektion meist vollen Effekt, während bei Erstgebärenden zwei Einspritzungen von je $1 \mathrm{ccm}$ der $2 \%$ igen Lösung mit einem Intervall von etwa drei Stunden die heftigsten Schmerzen beseitigen. Einen Einfluß auf die Geburtsdauer durch Schwächung der Wehen habe ich nicht beobachtet, auch wurden alle Kinder lebensfrisch geboren. Nur vermeide man, die Injektionen in der Austreibungsperiode auszuführen, weil die Wehen alsdann so wenig empfunden werden, daß die Kreißende nicht energisch mitpreßt. -
Da das Pantopon auch nach der Operation zur Schmerzlinderung ausreichend in Anwendung gezogen wurde und durchweg jede Kranke am Abend des Operationstages Pantopon 0,02 subkutan als Schlafmittel erhielt, konnten wir den Einfluß des Pantopons auf das postoperative Befinden der Kranken gut studieren. Ebenso wie Heimann bereits hervorgehoben hat, zeigten auch unsere Kranken keine nachteiligen Erscheinungen nach der Injektion. Die schlafmachende Wirkung stand der des Morphiums nicht nach. Da weder vor noch nach der Operation Morphium verabreicht wurde, blieb jede Alteration der Darmtätigkeit aus. An einer Serie von 30 genauer beobachteten Fällen gingen die Blähungen durchschnittlich 32 Stunden nach der Operation ab. Ich möchte die Abkürzung der von den Kranken so sehr empfundenen Flatulenzbeschwerden in erster Linie auf die ausschließliche Verwendung des Pantopons zurückführen.

Klinische Erfahrungen tragen häufig den Stempel des Subjektiven. Zahlreiche Untersuchungsreihen verschiedener Beobachter müssen diesen Fehler ausmerzen. Ich bin überzeugt, daß sie unsere günstigen Erfahrungen, die an der Hand eines nicht geringen klinischen Materiales gewonnen sind, bestätigen werden.

Zusammenfassung. Das Pantopon (Sahli) ist ein natürliches Kombinationsnarkotikum und eignet sich deshalb sehr gut zur Unterstützung der Allgemeinnarkose. Zwei subkutane Injektionen von $1 \mathrm{ccm}$ der fertigen, sterilen $2 \%$ igen Lösung (F. Hoffmann, La Roche \& Co.) führen in einem Drittel der nach Morphium-Skopolamin nötigen Zeit mit Aether eine tiefe Narkose herbei. Die erste Injektion wird 1,2 Stunden, die zweite $1 / 2$ Stunde vor Beginn der Narkose ausgeführt. Unter der Geburt läßt sich durch Pantopon ohne Schaden für Mutter und Kind eine gute Herabsetzung der Wehenschmerzen erzielen. Da auch die glatte Muskulatur des Darmes nicht gelähmt wird, gehen in der Rekonvaleszenz schon sehr frühzeitig die ersten Blähungen ab. 